\title{
Some Relations between Admissible Monomials for the Polynomial Algebra
}

\author{
Mbakiso Fix Mothebe ${ }^{1}$ and Lafras Uys ${ }^{2}$ \\ ${ }^{1}$ Department of Mathematics, University of Botswana, Private Bag 00704, Gaborone, Botswana \\ ${ }^{2}$ African Institute for Mathematical Sciences, 6 Melrose Road, Muizenberg, Cape Town, South Africa \\ Correspondence should be addressed to Mbakiso Fix Mothebe; mothebemf@mopipi.ub.bw
}

Received 14 April 2015; Accepted 5 July 2015

Academic Editor: Ram N. Mohapatra

Copyright (C) 2015 M. F. Mothebe and L. Uys. This is an open access article distributed under the Creative Commons Attribution License, which permits unrestricted use, distribution, and reproduction in any medium, provided the original work is properly cited.

Let $\mathbf{P}(n)=\mathbb{F}_{2}\left[x_{1}, \ldots, x_{n}\right]$ be the polynomial algebra in $n$ variables $x_{i}$, of degree one, over the field $\mathbb{F}_{2}$ of two elements. The mod-2 Steenrod algebra $\mathscr{A}$ acts on $\mathbf{P}(n)$ according to well known rules. A major problem in algebraic topology is of $\operatorname{determining} \mathscr{A}^{+} \mathbf{P}(n)$, the image of the action of the positively graded part of $\mathscr{A}$. We are interested in the related problem of determining a basis for the quotient vector space $\mathbf{Q}(n)=\mathbf{P}(n) / \mathscr{A}^{+} \mathbf{P}(n)$. $\mathbf{Q}(n)$ has been explicitly calculated for $n=1,2,3,4$ but problems remain for $n \geq 5$. Both $\mathbf{P}(n)=\bigoplus_{d>0} \mathbf{P}^{d}(n)$ and $\mathbf{Q}(n)$ are graded, where $\mathbf{P}^{d}(n)$ denotes the set of homogeneous polynomials of degree $d$. In this paper, we show that if $u=x_{1}^{m_{1}} \cdots x_{n-1}^{m_{n-1}} \in \mathbf{P}^{d^{\prime}}(n-1)$ is an admissible monomial (i.e., $u$ meets a criterion to be in a certain basis for $\mathbf{Q}(n-1)$ ), then, for any pair of integers $(j, \lambda), 1 \leq j \leq n$, and $\lambda \geq 0$, the monomial $h_{j}^{\lambda}(u)=x_{1}^{m_{1}} \cdots x_{j-1}^{m_{j-1}} x_{j}^{2^{\lambda-1}} x_{j+1}^{m_{j}} \cdots x_{n}^{m_{n-1}} \in \mathbf{P}^{d^{\prime}+\left(2^{\lambda}-1\right)}(n)$ is admissible. As an application we consider a few cases when $n=5$.

\section{Introduction}

For $n \geq 1$ let $\mathbf{P}(n)$ be the mod-2 cohomology group of the $n$ fold product of $\mathbb{R} P^{\infty}$ with itself. Then $\mathbf{P}(n)$ is the polynomial algebra

$$
\mathbf{P}(n)=\mathbb{F}_{2}\left[x_{1}, \ldots, x_{n}\right]
$$

in $n$ variables $x_{i}$, each of degree 1 , over the field $\mathbb{F}_{2}$ of two elements.

The mod-2 Steenrod algebra $\mathscr{A}$ is the graded associative algebra generated over $\mathbb{F}_{2}$ by symbols $\mathrm{Sq}^{i}$ for $i \geq 0$, called Steenrod squares subject to the Adem relations [1] and $\mathrm{Sq}^{0}=$ 1. Let $\mathbf{P}^{d}(n)$ denote the homogeneous polynomials of degree $d$. The action of the Steenrod squares $\mathrm{Sq}^{i}: \mathbf{P}^{d}(n) \rightarrow \mathbf{P}^{d+i}(n)$ is determined by the formula

$$
\mathrm{Sq}^{i}(u)= \begin{cases}u, & i=0, \\ u^{2}, & \operatorname{deg}(u)=i, \\ 0, & \operatorname{deg}(u)<i\end{cases}
$$

and the Cartan formula

$$
\mathrm{Sq}^{i}(u v)=\sum_{r=0}^{i} \mathrm{Sq}^{r}(u) \mathrm{Sq}^{i-r}(v)
$$

A polynomial $u \in \mathbf{P}^{d}(n)$ is said to be hit if it is in the image of the action of $\mathscr{A}$ on $\mathbf{P}(n)$, that is, if

$$
u=\sum_{i>0} \mathrm{Sq}^{i}\left(u_{i}\right),
$$

for some $u_{i} \in \mathbf{P}(n)$ of degree $d-i$. Let $\mathscr{A}^{+} \mathbf{P}(n)$ denote the subspace of all hit polynomials. The problem of determining $\mathscr{A}^{+} \mathbf{P}(n)$ is called the hit problem and has been studied by several authors [2-4]. We are interested in the related problem of determining a basis for the quotient vector space

$$
\mathbf{Q}(n)=\frac{\mathbf{P}(n)}{\mathscr{A}^{+} \mathbf{P}(n)}
$$


which has also been studied by several authors [5-8]. Some of the motivation for studying these problems is mentioned in [9]. It stems from the Peterson conjecture proved in [4] and various other sources $[10,11]$.

The following result is useful for determining $\mathscr{A}$ generators for $\mathbf{P}(n)$. Let $\alpha(m)$ denote the number of digits 1 in the binary expansion of $m$.

In $[4$, Theorem 1$]$, Wood proved the following.

Theorem 1 (Wood [4]). Let $u \in \mathbf{P}(n)$ be a monomial of degree d. If $\alpha(n+d)>n$, then $u$ is hit.

Thus, $\mathbf{Q}^{d}(n)$ is zero unless $\alpha(n+d) \leq n$ or, equivalently, unless $d$ can be written in the form $d=\sum_{i=1}^{n}\left(2^{\lambda_{i}}-1\right)$, where $\lambda_{i} \geq 0$. Thus, $\mathbf{Q}^{d}(n) \neq 0$ only if $\mathbf{P}^{d}(n)$ contains monomials $v=x_{1}^{2^{\lambda_{1}}-1} \cdots x_{n}^{2^{\lambda_{n}}-1}$ called spikes. For convenience, we will assume that $\lambda_{1} \geq \lambda_{2} \geq \cdots \geq \lambda_{n} \geq 0$. We, in addition, will consider a special one when $\lambda_{1} \geq \lambda_{2} \geq \cdots \geq \lambda_{s} \geq 0$ and $\lambda_{j-1}=\lambda_{j}$ only if $j=s$ or $\lambda_{j+1}=0$. In this case $v$ is called a minimal spike.

$\mathbf{Q}(n)$ has been explicitly calculated by Peterson [8] for $n=$ 1,2 , by Kameko in his thesis [5] for $n=3$, and independently by Kameko [6] and Sum [7] for $n=4$. In this work we will, unless otherwise stated, be concerned with a basis for $\mathbf{Q}(n)$ consisting of "admissible monomials," as defined below. Thus, when we write $u \in \mathbf{Q}^{d}(n)$ we mean that $u$ is an admissible monomial of degree $d$.

We define what it means for a monomial $b=x_{1}^{e_{1}} \cdots x_{n}^{e_{n}} \in$ $\mathbf{P}(n)$ to be admissible. Write $e_{i}=\sum_{j \geq 0} \alpha_{j}\left(e_{i}\right) 2^{j}$ for the binary expansion of each exponent $e_{i}$. The expansions are then assembled into a matrix $\beta(b)=\left(\alpha_{j}\left(e_{i}\right)\right)$ of digits 0 or 1 with $\alpha_{j}\left(e_{i}\right)$ in the $(i, j)$ th position of the matrix.

We then associate with $b$ two sequences

$$
\begin{aligned}
& w(b)=\left(w_{0}(b), w_{1}(b), \ldots, w_{j}(b), \ldots\right), \\
& e(b)=\left(e_{1}, e_{2}, \ldots, e_{n}\right),
\end{aligned}
$$

where $w_{j}(b)=\sum_{i=1}^{n} \alpha_{j}\left(e_{i}\right)$ for each $j \geq 0 . w(b)$ is called the weight vector of the monomial $b$ and $e(b)$ is called the exponent vector of the monomial $b$.

Given two sequences $p=\left(u_{0}, u_{1}, \ldots, u_{l}, 0, \ldots\right)$ and $q=$ $\left(v_{0}, v_{1}, \ldots, v_{l}, 0, \ldots\right)$, we say $p<q$ if there is a positive integer $k$ such that $u_{i}=v_{i}$ for all $i<k$ and $u_{k}<v_{k}$. We are now in a position to define an order relation on monomials.

Definition 2. Let $a, b$ be monomials in $\mathbf{P}(n)$. We say that $a<b$ if one of the following holds:

(1) $w(a)<w(b)$

(2) $w(a)=w(b)$ and $e(a)<e(b)$.

Note that the order relation on the set of sequences is the lexicographical one.
Following Kameko [5] we define the following.

Definition 3. A monomial $b \in \mathbf{P}(n)$ is said to be inadmissible if there exist monomials $b_{1}, b_{2}, \ldots, b_{r} \in \mathbf{P}(n)$ with $b_{j}<b$ for each $j, 1 \leq j \leq r$, such that

$$
b \equiv\left(\sum_{j=1}^{r} b_{j}\right) \bmod \mathscr{A}^{+} \mathbf{P}(n) .
$$

$b$ is said to be admissible if it is not inadmissible.

Clearly the set of all admissible monomials in $\mathbf{P}(n)$ form a basis for $\mathbf{Q}(n)$.

Let $u=x_{1}^{m_{1}} \cdots x_{n-1}^{m_{n-1}} \in \mathbf{P}(n-1)$ be a monomial of degree $d^{\prime}$. Given any pair of integers $(j, \lambda), 1 \leq$ $j \leq n, \lambda \geq 0$, we will write $h_{j}^{\lambda}(u)$ for the monomial $x_{1}^{m_{1}} \cdots x_{j-1}^{m_{j-1}} x_{j}^{2^{\lambda}-1} x_{j+1}^{m_{j}} \cdots x_{n}^{m_{n-1}} \in \mathbf{P}^{d^{\prime}+\left(2^{\lambda}-1\right)}(n)$. Our main result is the following.

Theorem 4. Let $u \in \mathbf{P}(n-1)$ be a monomial of degree $d^{\prime}$, where $\alpha\left(d^{\prime}+n-1\right) \leq n-1$. If $u$ is admissible, then, for each pair of integers $(j, \lambda), 1 \leq j \leq n, \lambda \geq 0, h_{j}^{\lambda}(u)$ is admissible.

Our proof of Theorem 4 is deferred until Section 3.

As a corollary to Theorem 4, suppose that $\lambda$ is fixed so that $d^{\prime}$ is also fixed. Let $x_{1}^{2^{\lambda_{1}}-1} \cdots x_{n-2}^{2^{\lambda_{n-2}-1}}$ be the minimal spike of degree $d^{\prime}$. If $\lambda>\lambda_{1}$, then consider the following.

Corollary 5. $\mathbf{Q}^{d}(n)$ has a subspace isomorphic to $\bigoplus_{i=1}^{n} \mathbf{Q}^{d^{\prime}}(n-$ $1)$.

As our main application of the theorem we consider a few cases when $n=5$. The relevant result in this case is Theorem 6 stated below. To explain Table 1 we recall that, given an integer $d$ such that $\alpha(n+d) \leq n$, we let

$$
C(n, d)=\left\{\lambda \geq 0 \mid \alpha\left(d-\left(2^{\lambda}-1\right)+n-1\right) \leq n-1\right\} .
$$

Then given any explicit admissible monomial basis for $\mathbf{Q}(n-$ 1) one may compute $\mathscr{L} B(n, d)$, the dimension of the subspace of $\mathbf{Q}^{d}(n)$ generated by all monomials of the form $h_{j}^{\lambda}(u) \epsilon$ $\mathbf{P}^{d}(n), \lambda \in C(n, d)$ and $u \in \mathbf{Q}^{d-\left(2^{\lambda}-1\right)}(n-1)$. In general $\mathscr{L} B(n, d) \leq \operatorname{dim}\left(\mathbf{Q}^{d}(n)\right)$.

In [7] Sum gives an explicit admissible monomial basis for $\mathbf{Q}(4)$. In this paper we make use of his results to compute $\mathscr{L} B(5, d), 1 \leq d \leq 30$, and compare these values with $\operatorname{dim}\left(\mathbf{Q}^{d}(5)\right)$ in the given range. The results are given in Table 1. The table is incomplete as not all values for $\operatorname{dim}\left(\mathbf{Q}^{d}(5)\right)$ are known in the given range. While in general it is true that $\mathscr{L} B(5, d) \leq \operatorname{dim}\left(\mathbf{Q}^{d}(5)\right)$, there are cases, even nontrivial, where equality holds. This is demonstrated with the aid of known results for $\operatorname{dim}\left(\mathbf{Q}^{d}(5)\right)$ (cited in Table 1) but we will show later that the same conclusions can be reached independently.

Theorem 6. Table 1 gives lower bounds, $\mathscr{L} B(5, d)$, for the dimension of $\mathbf{Q}^{d}(5), 1 \leq d \leq 30$. 
TABLE 1

\begin{tabular}{|c|c|c|c|}
\hline$d$ & $\operatorname{dim}\left(\mathbf{Q}^{d}(5)\right)$ & Reference & $\mathscr{L} B(5, d)$ \\
\hline 1 & 5 & & 5 \\
\hline 2 & 10 & & 10 \\
\hline 3 & 25 & & 25 \\
\hline 4 & 45 & [12] & 45 \\
\hline 5 & 46 & & 46 \\
\hline 6 & 74 & & 74 \\
\hline 7 & 110 & & 110 \\
\hline 8 & 174 & {$[13]$} & 174 \\
\hline 9 & 191 & & 191 \\
\hline 10 & 280 & & 275 \\
\hline 11 & 315 & [14] & 313 \\
\hline 12 & 190 & [12] & 190 \\
\hline 13 & - & & 246 \\
\hline 14 & - & & 295 \\
\hline 15 & 432 & [15] & 384 \\
\hline 16 & - & & 414 \\
\hline 17 & - & & 538 \\
\hline 18 & - & & 673 \\
\hline 19 & - & & 778 \\
\hline 20 & - & & 591 \\
\hline 21 & - & & 741 \\
\hline 22 & - & & 780 \\
\hline 23 & - & & 916 \\
\hline 24 & - & & 908 \\
\hline 25 & - & & 974 \\
\hline 26 & 1024 & [16] & 985 \\
\hline 27 & 315 & & 313 \\
\hline 28 & 480 & {$[12]$} & 480 \\
\hline 29 & - & & 450 \\
\hline 30 & - & & 763 \\
\hline
\end{tabular}

While this approach remains to be explored, in general these test results suffice for our purpose in this paper and we hope to make a more general account in subsequent work. We are thus only required to prove Theorem 4 .

Our work is organized as follows. In Section 2, we recall some results on admissible monomials and hit monomials in $\mathbf{P}(n)$. In Section 3, we prove Theorem 4. We conclude with an application of the theorem in Section 4.

\section{Preliminaries}

In this section we recall some results in Kameko [17] and Singer [3] on admissible monomials and hit monomials in $\mathbf{P}(n)$.

The following theorem has been used to great effect by Kameko and Sum in computing a basis for $\mathbf{Q}(3)$ and $\mathbf{Q}(4)$, respectively.

Theorem 7 (Kameko [5] and Sum [18]). Let a, $b$ be monomials in $\mathbf{P}(n)$ such that $w_{j}(a)=0$ for $j>r>0$. If $b$ is inadmissible, then $a b^{2^{r}}$ is also inadmissible.
Up to permutation of representatives weight order provides a total order relation amongst spikes in a given degree.

It is easy to show that a spike $v=x_{1}^{2^{\lambda_{1}}-1} \cdots x_{n}^{2^{\lambda_{n}}-1} \in \mathbf{P}^{d}(n)$ is a minimal spike if its weight order is minimal with respect to other spikes of degree $d$. We say $v$ is a maximal spike if its weight order is maximal with respect to other spikes of degree $d$. In [17, Theorem 4.2] Kameko proved the following.

Theorem 8 (Kameko). Let $d$ be a positive integer and let $v$ be the minimal spike of degree $2 d+n$. Define a linear mapping, $f: \mathbf{P}^{d}(n) \rightarrow \mathbf{P}^{2 d+n}(n)$, by

$$
f\left(x_{1}^{m_{1}} \cdots x_{n}^{m_{n}}\right)=x_{1}^{2 m_{1}+1} \cdots x_{n}^{2 m_{n}+1} .
$$

If $w_{0}(v)=n$, then $f$ induces an isomorphism $f_{*}: \mathbf{Q}^{d}(n) \rightarrow$ $\mathbf{Q}^{2 d+n}(n)$.

From Wood's theorem and the above result of Kameko the problem of determining $\mathscr{A}$-generators for $\mathbf{P}(n)$ is reduced to the cases for which $w_{0}(v) \leq n-1$ whenever $v$ is a minimal spike of a given degree $d$.

We recall the following result of Singer on hit polynomials in $\mathbf{P}(n)$. In [3, Theorem 1.2], Singer proved the following.

Theorem 9 (Singer). Let $b \in \mathbf{P}(n)$ be a monomial of degree $d$, where $\alpha(n+d) \leq n$. Let $v$ be the minimal spike of degree $d$. If $w(b)<w(v)$, then $b$ is hit.

We will require the following stronger version of Theorem 9. Let $b$ be a monomial of degree $d$. For $l>0$ define $d_{l}(b)$ to be the integer $d_{l}(b)=\sum_{j \geq l} w_{j}(b) 2^{j-l}$.

In [2, Theorem 1.2], Silverman proved the following.

Theorem 10 (Silverman). Let $b \in \mathbf{P}(n)$ be a monomial of degree $d$, where $\alpha(n+d) \leq n$. Let $v$ be the minimal spike of degree $d$. If $d_{l}(b)>d_{l}(v)$ for some $l \geq 1$, then $b$ is hit.

Finally we note that for any element $\mathrm{Sq}^{k} \in \mathscr{A}^{+}$and any polynomial $u \in \mathbf{P}(n)$ we have

$$
\mathrm{Sq}^{k 2^{\lambda}}\left(u^{2^{\lambda}}\right)=\left(\mathrm{Sq}^{k}(u)\right)^{2^{\lambda}}
$$

for a given $\lambda \geq 0$.

\section{Proof of Theorem 4}

In this section we prove Theorem 4. Our main observation in this work is made in terms of pairs of integers $j, \lambda, 1 \leq j \leq n$, $\lambda \geq 0$, which determine a monomial $h_{j}^{\lambda}(u) \in \mathbf{P}^{d^{\prime}+\left(2^{\lambda}-1\right)}(n)$ once a monomial $u \in \mathbf{P}^{d^{\prime}}(n-1)$ is given. We first note that if $u=x_{1}^{e_{1}} \cdots x_{n-1}^{e_{n-1}}$, then $h_{n}^{\lambda}(u)=x_{1}^{e_{1}} \cdots x_{n-1}^{e_{n-1}} x_{n}^{2^{\lambda}-1}$ while, for any other value of $j, h_{j}^{\lambda}(u)$ is a permutation of $h_{n}^{\lambda}(u)$ that replaces the exponent of $x_{j}$ by $2^{\lambda}-1$ and those for $x_{j+i}, n-j \geq i \geq 1$, by $e_{j+i-1}$. We may therefore use permutation notation in place of $j$. Let

$$
S(n)=\left\{\sigma \in S_{n} \mid \sigma(r)<\sigma(s) \text { if } r<s<n\right\} .
$$


Then $|S(n)|=n$ and for each integer $j, 1 \leq j \leq n$, there exists $\sigma \in S(n)$ such that $h_{j}^{\lambda}\left(x_{1}^{e_{1}} \cdots x_{n-1}^{e_{n-1}}\right)=x_{\sigma(1)}^{e_{1}} \cdots x_{\sigma(n-1)}^{e_{n-1}} x_{\sigma(n)}^{2^{\lambda}-1}$ whenever $x_{1}^{e_{1}} \cdots x_{n-1}^{e_{n-1}} \in \mathbf{P}^{d^{\prime}}(n-1)$. For convenience of presentation we will write $h_{\sigma}^{\lambda}\left(x_{1}^{e_{1}} \cdots x_{n-1}^{e_{n-1}}\right)$ in place of $h_{j}^{\lambda}\left(x_{1}^{e_{1}} \cdots x_{n-1}^{e_{n-1}}\right)$.

Proof of Theorem 4. Suppose that $u=x_{1}^{e_{1}} \cdots x_{n-1}^{e_{n-1}} \in \mathbf{P}(n-1)$ is a monomial of degree $d^{\prime}$, where $\alpha\left(d^{\prime}+n-1\right) \leq n-1$. Let $\lambda \geq 0$ be an integer. We must show that, for any permutation $\sigma \in S(n), h_{\sigma}^{\lambda}(u)$ is admissible whenever $u$ is admissible. Put $d=d^{\prime}+2^{\lambda}-1$.

We first note that, for each $\sigma \in S(n)$, every monomial in $h_{\sigma}^{\lambda}(\mathbf{P}(n-1))$ is of the form

$$
x_{\sigma(1)}^{m_{1}} \cdots x_{\sigma(n-1)}^{m_{n-1}} x_{\sigma(n)}^{2^{\lambda}-1} .
$$

Thus, if $\mathrm{Sq}^{k} \in \mathscr{A}^{+}$and $w$ is a monomial in $\mathbf{P}^{d-k}(n)$ for which $\mathrm{Sq}^{k}(w)$ contains a term belonging to $h_{\sigma}^{\lambda}\left(\mathbf{P}^{d^{\prime}}(n-1)\right)$, then $w$ must be of the form

$$
x_{\sigma(1)}^{e_{1}} \cdots x_{\sigma(n-1)}^{e_{n-1}} x_{\sigma(n)}^{2^{\lambda}-1},
$$

for some $x_{1}^{e_{1}} \cdots x_{n-1}^{e_{n-1}} \in \mathbf{P}^{d^{\prime}-k}(n-1)$. This must be the case since for any pair of positive integers $\lambda, i$ there exists no integer $t$ such that $\mathrm{Sq}^{i}\left(x^{t}\right)=x^{2^{\lambda}-1}$. By the Cartan formula $\mathrm{Sq}^{k}(w)$ may be written in the form

$$
\begin{aligned}
& \mathrm{Sq}^{k}\left(x_{\sigma(1)}^{e_{1}} \cdots x_{\sigma(n-1)}^{e_{n-1}}\right) x_{\sigma(n)}^{2^{\lambda}-1} \\
& \quad+\sum_{t \geq 1} \mathrm{Sq}^{k-t}\left(x_{\sigma(1)}^{e_{1}} \cdots x_{\sigma(n-1)}^{e_{n-1}}\right) \operatorname{Sq}^{t}\left(x_{\sigma(n)}^{2^{\lambda}-1}\right) .
\end{aligned}
$$

For a given permutation $\sigma \in S(n)$ write

$$
\mathbf{P}^{d}(n)=\mathbf{P}^{(d, \sigma)}(n) \bigoplus h_{\sigma}^{\lambda}\left(\mathbf{P}^{d^{\prime}}(n-1)\right),
$$

where $\mathbf{P}^{(d, \sigma)}(n)$ is the complement of $h_{\sigma}^{\lambda}\left(\mathbf{P}^{d^{\prime}}(n-1)\right)$ in $\mathbf{P}^{d}(n)$ and let $\pi_{\sigma}$ denote the projection

$$
\pi_{\sigma}: \mathbf{P}^{d}(n) \longrightarrow h_{\sigma}^{\lambda}\left(\mathbf{P}^{d^{\prime}}(n-1)\right)
$$

of $\mathbf{P}^{d}(n)$ onto $h_{\sigma}^{\lambda}\left(\mathbf{P}^{d^{\prime}}(n-1)\right)$ with respect to this splitting.

The assignment,

$$
\begin{aligned}
h_{\sigma}^{\lambda} & : \mathrm{Sq}^{k}\left(x_{1}^{e_{1}} \cdots x_{n-1}^{e_{n-1}}\right) \\
& \longmapsto \mathrm{Sq}^{k}\left(x_{\sigma(1)}^{e_{1}} \cdots x_{\sigma(n-1)}^{e_{n-1}}\right) x_{\sigma(n)}^{2^{\lambda}-1},
\end{aligned}
$$

gives a bijection between

$$
\begin{gathered}
\mathscr{A}^{+} \mathbf{P}(n-1) \cap \mathbf{P}^{d^{\prime}}(n-1), \\
\pi_{\sigma}\left(\mathscr{A}^{+} \mathbf{P}(n) \cap \mathbf{P}^{d}(n)\right) .
\end{gathered}
$$

Thus, for each $\sigma \in S(n)$, we can think of $h_{\sigma}^{\lambda}$ as having an inverse

$$
g_{\sigma}^{\lambda}: \pi_{\sigma}\left(\mathbf{P}^{d}(n)\right) \longrightarrow \mathbf{P}^{d^{\prime}}(n-1)
$$

Now, let $u \in \mathbf{Q}^{d^{\prime}}(n-1)$. Then, $h_{\sigma}^{\lambda}(u)$ is an element of $h_{\sigma}^{\lambda}\left(\mathbf{P}^{d^{\prime}}(n-1)\right)$. Thus, any hit polynomial that has $h_{\sigma}^{\lambda}(u)$ as a term is a sum of polynomial expressions of the form (14). Proceeding by contraposition, suppose that $h_{\sigma}^{\lambda}(u)$ is inadmissible; that is, it is not an element of $\mathbf{Q}^{d}(n)$. Then we can find a polynomial in which modulo image of the action, $h_{\sigma}^{\lambda}(u)$, is a sum of monomials of the form $\left(x_{\sigma(1)}^{m_{1}} \cdots x_{\sigma(n-1)}^{m_{n-1}}\right) x_{\sigma(n)}^{2^{\lambda}-1}$ plus error terms $\mathrm{Sq}^{k-t}\left(x_{\sigma(1)}^{e_{1}} \cdots x_{\sigma(n-1)}^{e_{n-1}}\right) \mathrm{Sq}^{t}\left(x_{\sigma(n)}^{2^{\lambda}-1}\right)$ all of order lower than that of $h_{\sigma}^{\lambda}(u)$. Since in this event the error terms are of no consequence (in terms of determining whether $u$ is admissible or not) we may as well restrict ourselves to that part of the polynomial which consists of terms belonging to $\pi_{\sigma}\left(\mathbf{P}^{d}(n)\right)$, that is, to the image of $h_{\sigma}^{\lambda}$. But the mapping $h_{\sigma}^{\lambda}$ is injective and preserves the order of monomials, so by considering its inverse $g_{\sigma}^{\lambda}$ we see that $u$ must also be inadmissible.

Proof of Corollary 5. We must show that if $\lambda$ is fixed and is greater than $\lambda_{1}$, then for any permutation $\sigma \in S(n)$ we have $u \in \mathbf{Q}^{d^{\prime}}(n-1)$ if and only if $h_{\sigma}^{\lambda}(u) \in \mathbf{Q}^{d}(n)$, or, by virtue of Theorem 4 , that we have $u$ admissible whenever $h_{\sigma}^{\lambda}(u)$ is admissible. But any hit polynomial which has $h_{\sigma}^{\lambda}(u)$ as a term is generated by polynomial expressions of the form (14) so it suffices to show that an error term in each such polynomial expression,

$$
\mathrm{Sq}^{k-t}\left(x_{\sigma(1)}^{e_{1}} \cdots x_{\sigma(n-1)}^{e_{n-1}}\right) \operatorname{Sq}^{t}\left(x_{\sigma(n)}^{2^{\lambda}-1}\right),
$$

is either hit or of lower weight order than that of $h_{\sigma}^{\lambda}(u)$. But if $v=x_{1}^{2^{\lambda_{1}}-1} \cdots x_{n-2}^{2^{\lambda_{n-2}-1}}$ is the minimal spike of degree $d^{\prime}, \lambda>$ $\lambda_{1}$, and $b$ is a term in a polynomial expression of the form (20), then $d_{l}(b)>d_{l}(v)$ for $l=\lambda_{1}$ and so by Theorem $10 b$ is hit. The proof is then completed by noting that if $\lambda>\lambda_{1}$, then the subspace of $\mathbf{P}^{d}(n)$ spanned by $\bigcup_{\sigma} h_{\sigma}^{\lambda}\left(\mathbf{P}^{d^{\prime}}(n-1)\right)$ is a direct summand $\bigoplus_{\sigma} h_{\sigma}^{\lambda}\left(\mathbf{P}^{d^{\prime}}(n-1)\right)$ of $\mathbf{P}^{d}(n)$ isomorphic to $\bigoplus_{j=1}^{n}\left(\mathbf{P}^{d^{\prime}}(n-1)\right)$ and that the respective subspace of $\mathbf{Q}^{d}(n)$ with basis, the union over all $\sigma \in S(n)$ of the sets $\left\{h_{\sigma}^{\lambda}(u) \mid u \epsilon\right.$ $\left.\mathbf{Q}^{d^{\prime}}(n-1)\right\}$ inherits this splitting.

\section{Application of Theorem 4}

We have already applied Theorem 4 to derive the table of Theorem 6 . The table can be extended to all $d \geq 0$ but in this work we have limited ourselves to $d \leq 30$. The purpose of this restriction is to show that, besides the cases, $1 \leq d \leq 9$, we also have $\operatorname{dim}\left(\mathbf{Q}^{d}(5)\right)=\mathscr{L} B(5, d)$, when $d=12$ and $d=28$. We also compute $\operatorname{dim}\left(\mathbf{Q}^{d}(5)\right)$ for $d=10$ and $d=11$.

To prove all this we require some preliminary observations. For each $r, 1 \leq r \leq n$, let

$$
\mathbf{X}(r)=\operatorname{Span}\left\{x_{1}^{m_{1}} \cdots x_{r}^{m_{r}} \in \mathbf{P}(r) \mid m_{1} m_{2} \cdots m_{\mathrm{r}} \neq 0\right\} .
$$

Then $\mathbf{X}(r)$ is an $\mathscr{A}$-submodule of $\mathbf{P}(r)$. Let

$$
\mathbf{W}(r)=\frac{\mathbf{X}(r)}{\mathscr{A}^{+} \mathbf{X}(r)} .
$$


Then we have a direct sum decomposition

$$
\mathbf{Q}(n) \cong \bigoplus_{r=1}^{n} \bigoplus_{k=1}^{\left(\begin{array}{l}
n \\
r
\end{array}\right)} \mathbf{W}(r) .
$$

Thus, we have the following.

For any integer $d>0$,

$$
\operatorname{dim}\left(\mathbf{Q}^{d}(n)\right)=\sum_{r=1}^{n}\left(\begin{array}{l}
n \\
r
\end{array}\right) \operatorname{dim}\left(\mathbf{W}^{d}(r)\right) .
$$

If we were to compute $\operatorname{dim}\left(\mathbf{Q}^{d}(n)\right)$ by an inductive procedure on the number of variables, then we would only be required to compute $\operatorname{dim}\left(\mathbf{W}^{d}(n)\right)$ in proceeding from the case $n-1$ to $n$. Apart from spikes all subsequent references to monomials will mean those belonging to $\mathbf{X}(n)$ and, by virtue of Singer's Theorem 9, we will assume they are of weight order greater than or equal to that of the minimal spike. We note also that any variation between $\operatorname{dim}\left(\mathbf{Q}^{d}(n)\right)$ and $\mathscr{L} B(n, d)$ is dependant only on $\mathbf{X}^{d}(n)$, since, for any permutation $\sigma \in$ $S(n)$, whenever $u \in \mathbf{Q}^{d}(n-1)$, then choosing $\lambda=0$, we have $h_{\sigma}^{\lambda}(u) \in \mathbf{Q}^{d}(n)$.

That $\operatorname{dim}\left(\mathbf{Q}^{d}(5)\right)=\mathscr{L} B(5, d), 1 \leq d \leq 9, d=12$ and $d=28$ will be consequent from the following results.

Let $d \geq 0$ and suppose that $\alpha(d+n) \leq n$. Suppose there is a spike $v=x_{1}^{2^{\lambda_{1}}-1} \cdots x_{n-1}^{2^{\lambda_{n-1}-1}} \in \mathbf{P}^{d}(n)$ for which $w_{0}(v)=$ $n-1$. We will say that a monomial $a=x_{1}^{m_{1}} \cdots x_{n}^{m_{n}} \in \mathbf{X}^{d}(n)$ of weight order greater than or equal to that of $v$ is associated with $v$ if, for some pair $(i, j)$, we have $m_{j}=2^{\lambda_{i}}-1$ and is strongly associated with $v$ if $a$ is associated with $v$ and if in addition $m_{j} \leq 2^{n-2}-1$ for some $j$. Note that a monomial $a$ is associated with a spike $v$ if $a=h_{j}^{\lambda_{i}}(u)$ for some $\lambda_{i}$ and some monomial $u \in \mathbf{X}(n-1)$.

There are cases, dependent on $d$, where every monomial in $\mathbf{X}^{d}(n)$ is strongly associated with some spike $v$ of degree $d$. In particular we have the following.

Lemma 11. Let $d$ be an integer for which $\alpha(n+d) \leq n$ and let $a \in \mathbf{X}^{d}(n)$ be a monomial. Suppose that for each spike $v=$ $x_{1}^{2^{\lambda_{1}}-1} \cdots x_{n-1}^{2^{\lambda_{n-1}-1}}$ of degreed we have $\lambda_{n-1}>0, \lambda_{1} \leq n-1$, $\lambda_{i}-\lambda_{i+1} \leq 1$, and $\lambda_{1}-\lambda_{n-1} \leq 2$. Then $a$ is strongly associated with at least one of the spikes of degree $d$.

Proof. Suppose that every spike of degree $d$ satisfies the hypothesis of the lemma. We first show that if $a=$ $x_{1}^{m_{1}} \cdots x_{n}^{m_{n}} \in \mathbf{X}^{d}(n)$ with $w(a)=w(v)$ for some spike $v$ then $a$ is strongly associated with $v$. To see this represents the spike $v$ in matrix form. Without loss of generality assume that row 1 of the matrix has zero entries so that row $i+1$, column $j$ has entry $\alpha_{j}\left(2^{\lambda_{i}}-1\right), 1 \leq i \leq n-1$. Note that the columns are indexed by $j, 0 \leq j \leq n-2$. The matrix form of a monomial $a$ of the same weight order as $v$ may be obtained from the matrix form of $v$ by moving ones from the $n-1$ rows of $v$ vertically upwards or downwards to a different row including row 1 . In the worst case scenario we can move at most $n-2$ ones to row 1 from $n-2$ distinct rows of the matrix form of $v$. Thus, we must be left with at least one row with consecutive ones; that is, for any monomial obtained this way we must have $m_{j}=2^{\lambda_{i}}-1$ for some $j$. Since $\lambda_{1} \leq n-1$, we must have $m_{j} \leq 2^{n-2}-1$ for some $j$. Suppose that $w(a) \neq w(v)$ for any spike $v$ of degree $d$. It is easy to see that this is possible only if there is a spike $v$ for which $\lambda_{1}-\lambda_{n-1}=2$. We claim that we must have $m_{j}=2^{\lambda_{1}-1}-1$ for some $j$. In this case a monomial of higher order than $v$ can only be obtained by applying the splitting $2^{\lambda}+2^{\lambda}=2^{\lambda+1}$ to $2^{\lambda_{i}}$ where $\lambda_{i}=\lambda_{1}$. The proof is then completed by noting that $\lambda_{1}-1 \leq n-2$.

We are now in a position to show that $\operatorname{dim}\left(\mathbf{Q}^{d}(5)\right)=$ $\mathscr{L} B(5, d)$ when $1 \leq d \leq 9, d=12$, and $d=28$. We will make use of the following observation.

Suppose that $d$ is an integer for which the minimal spike $v$ of degree $d$ satisfies the condition $w_{o}(v)=n-1$. For each $t$, $1 \leq t \leq n$, let $f_{t}: \mathbf{P}^{d}(n) \rightarrow \mathbf{P}^{2 d+n-1}(n)$ be the linear mapping given on monomials by

$$
\begin{aligned}
f_{t} & \left(x_{1}^{e_{1}} \cdots x_{n}^{e_{n}}\right) \\
& =x_{1}^{2 e_{1}+1} \cdots x_{t-1}^{2 e_{t-1}+1} x_{t}^{2 e_{t}} x_{t+1}^{2 e_{t+1}+1} \cdots x_{n}^{2 e_{n}+1} .
\end{aligned}
$$

Then, ignoring monomials of order lower than that of the minimal spike of degree $2 d+n-1$, it is easy to see, by virtue of (10), that

$$
\begin{aligned}
\mathscr{A}^{+} \mathbf{P}(n) \cap \mathbf{P}^{d^{\prime}}(n) \\
=\left[\operatorname{Sq}^{1}(\mathbf{P}(n)) \cap \mathbf{P}^{d^{\prime}}(n)\right] \\
\oplus\left[\bigoplus_{t=1}^{n} f_{t}\left(\mathscr{A}^{+} \mathbf{P}(n) \cap \mathbf{P}^{d}(n)\right)\right],
\end{aligned}
$$

where $d^{\prime}=2 d+n-1$ and $\operatorname{Sq}^{1}(\mathbf{P}(n))$ is the image of the action of $\mathrm{Sq}^{1}$ on $\mathbf{P}(n)$. In other words the image of $\mathrm{Sq}^{k}, k>1$, is given by $\bigoplus_{t=1}^{n} f_{t}\left(\mathscr{A}^{+} \mathbf{P}(n) \cap \mathbf{P}^{d}(n)\right)$.

When $n=5$ then the spikes $x_{1}^{7} x_{2}^{7} x_{3}^{7} x_{4}^{7}, x_{1}^{15} x_{2}^{7} x_{3}^{3} x_{4}^{3}$, of degree 28, satisfy the hypothesis of Lemma 11 and each monomial of this degree is of the weight order of one of these spikes. Let $a=x_{1}^{m_{1}} x_{2}^{m_{2}} x_{3}^{m_{3}} x_{4}^{m_{4}} x_{5}^{m_{5}} \in \mathbf{X}^{28}(5)$. Then $m_{j}=3,7,15$ for some $j$ or equivalently we can find $\sigma \in S(5)$ and a monomial $u \in \mathbf{X}(4)$ such that $a=h_{\sigma}^{\lambda}(u)$ where $\lambda=2,3$, or 4 . To prove that $\operatorname{dim}\left(\mathbf{Q}^{28}(5)\right)=\mathscr{L} B(5,28)$, it is sufficient to show that whenever $a$ is admissible then we can find an admissible monomial $u \in \mathbf{X}(4)$ and a permutation $\sigma \in S(5)$ such that $h_{\sigma}^{\lambda}(u)=a(\lambda=2,3$, or 4$)$. Since $a$ is of the form $h_{\sigma}^{\lambda}(u)$ for some $u \in \mathbf{X}(4)$ it is sufficient to show that if $f$ is a hit polynomial that has $a$ as a term, then the error terms are either hit or of lower order than $a$. It is easy to see that if $a$ is of the weight order of the spike $x_{1}^{7} x_{2}^{7} x_{3}^{7} x_{4}^{7}$ and $\mathrm{Sq}^{k}(w)$ is a hit polynomial that has $a$ as a term, then all the error terms are of lower weight order than that of $a$. Clearly this suffices to show that every admissible monomial of the weight order of the spike $x_{1}^{7} x_{2}^{7} x_{3}^{7} x_{4}^{7}$ is of the form $h_{\sigma}^{3}(u)$ for some admissible monomial $u \in \mathbf{X}(4)$. 
That leaves us with the case where $a$ has weight order that of the minimal spike $v=x_{1}^{15} x_{2}^{7} x_{3}^{3} x_{4}^{3}$. In this case we can find $j, 1 \leq j \leq 5$, such that $m_{j}$ is equal to at least one $3,7,15$. If $m_{j}=15$ for some $j$, then the hypothesis of Corollary 5 is satisfied if we take $d^{\prime}=13$ and $\lambda=4$; hence, if $a$ is admissible then $a=h_{\sigma}^{4}(u)$ for some admissible monomial $u \in \mathbf{X}^{13}(4)$. We may therefore suppose that $m_{j} \neq 15$ for any $j$. We show that in all cases each element of $\mathscr{A}^{+} \mathbf{P}(5) \cap \mathbf{P}^{28}(5)$ that has $a$ as a term has error terms that consist of hit monomials. When $n=5$, then $2 d+n-1=28$ implies $d=12$ and the minimal spike when $d=12$ is $v=x_{1}^{7} x_{2}^{3} x_{3} x_{4}$. Since $w_{0}(v)=4$ we have

$$
\begin{aligned}
\mathscr{A}^{+} \mathbf{P}(5) & \cap \mathbf{P}^{28}(5) \\
= & {\left[\operatorname{Sq}^{1}(\mathbf{P}(5)) \cap \mathbf{P}^{28}(n)\right] } \\
\oplus & {\left[\bigoplus_{t=1}^{5} f_{t}\left(\mathscr{A}^{+} \mathbf{P}(5) \cap \mathbf{P}^{12}(5)\right)\right] . }
\end{aligned}
$$

Since $f_{t}$ preserves the order of monomials for each $t$ we may, by induction, assume that whenever $a \in \mathbf{X}^{12}(5)$ is admissible then we can find an admissible monomial $u \in \mathbf{X}(4)$ and a permutation $\sigma \in S(5)$ such that $h_{\sigma}^{\lambda}(u)=a(\lambda=1,2$, or 3$)$. Thus, we need only to consider the case when $a$ is a term in $\mathrm{Sq}^{1}(\mathbf{P}(5))$. Since $w_{0}(a)=w_{1}(a)=4$, we see that the error part of a hit polynomial that has $a$ as a term consists of monomials which are hit unless if in the matrix form of $a$ we have $\alpha_{o}\left(m_{i}\right)=\alpha_{1}\left(m_{i}\right)=0$ for some $i$. In this case $a$ is a permutation representative of one of $x_{1}^{7} x_{2}^{3} x_{3}^{3} x_{4}^{3} x_{5}^{12}$, $x_{1}^{7} x_{2}^{3} x_{3}^{3} x_{4}^{4} x_{5}^{11}$, or $x_{1}^{7} x_{2}^{7} x_{3}^{3} x_{4}^{3} x_{5}^{8}$. Consider the cases where $a$ is a permutation representative of one of $x_{1}^{7} x_{2}^{3} x_{3}^{3} x_{4}^{3} x_{5}^{12}$ or $x_{1}^{7} x_{2}^{3} x_{3}^{3} x_{4}^{4} x_{5}^{11}$. If we take $m_{j}=7$, then the error terms in a hit polynomial, $\mathrm{Sq}^{1}(w)$, that has $a=h_{j}^{3}(u)$ as a term are hit monomials. Finally it is easy to check that the case where $\mathrm{Sq}^{1}(\mathbf{P}(5))$ has an element which has a permutation representative of $x_{1}^{7} x_{2}^{7} x_{3}^{3} x_{4}^{3} x_{5}^{8}$ as a term is when such an element is a sum of hit polynomials in $\mathbf{P}^{28}(5)$. Such action makes no contribution (in terms of adding or deducting) to the basis of $\mathbf{Q}^{28}(5)$. A similar argument applies to monomials of weight order of the spike $x_{1}^{7} x_{2}^{3} x_{3} x_{4}$ of degree 12 which is also the base of our induction in the case $d=28$.

We now show that $\operatorname{dim}\left(\mathbf{Q}^{d}(5)\right)=\mathscr{L} B(5, d)$ when $1 \leq$ $d \leq 9$. There is nothing to show in the cases $1 \leq d \leq 4$ as $\operatorname{dim}\left(\mathbf{Q}^{d}(5)\right)=\mathscr{L} B(5, d)$ whenever $\mathbf{X}^{d}(5)=0$. In the case $d=5$ the spike $v=x_{1} x_{2} x_{3} x_{4} x_{5}$ is the only element in $\mathbf{W}^{5}(5)$. In the cases $d=6,7$ each monomial in $\mathbf{X}^{d}(5)$ is associated with one of the respective spikes $x_{1}^{3} x_{2} x_{3} x_{4}$ and $x_{1}^{3} x_{2}^{3} x_{3}, x_{1}^{3} x_{2} x_{3} x_{4} x_{5}$ and it is easy to verify that those of the form $h_{\sigma}^{\lambda}(u)$ for some $u \in \mathbf{W}(4)$ form basis for $\mathbf{W}^{6}(5)$ and $\mathbf{W}^{7}(5)$, respectively. Similarly in the cases $d=8,9$.

If $d=10$, then one can verify that in addition to the elements obtained from $\mathbf{W}(4)$ via the mappings $h_{\sigma}^{\lambda}$ the five monomials $x_{1} x_{2} x_{3}^{2} x_{4}^{2} x_{5}^{4}, x_{1} x_{2} x_{3}^{2} x_{4}^{4} x_{5}^{2}, x_{1} x_{2}^{2} x_{3} x_{4}^{2} x_{5}^{4}$, $x_{1} x_{2}^{2} x_{3} x_{4}^{4} x_{5}^{2}$, and $x_{1} x_{2}^{2} x_{3}^{4} x_{4} x_{5}^{2}$ are also admissible so that $\operatorname{dim}\left(\mathbf{Q}^{d}(5)\right)=280$. If $d=11$, then it is easy to show that in addition to the monomials generated from W(4) the two monomials $x_{1} x_{2} x_{3}^{2} x_{4}^{2} x_{5}^{5}, x_{1} x_{2}^{2} x_{3} x_{4}^{2} x_{5}^{5}$ also belong to $\mathbf{W}^{11}(5)$ or are also admissible so that $\operatorname{dim}\left(\mathbf{Q}^{11}(5)\right)=315$.

Finally we note that if $v \in \mathbf{P}^{d}(n)$ is a spike that satisfies the hypothesis of Lemma 11 and is one for which $\lambda_{1}-\lambda_{n-1} \leq 1$, then $v$ is the maximal spike and $w(v) \geq w(a)$ for every monomial $a \in \mathbf{P}^{d}(n)$. In these cases monomials $a \in \mathbf{X}^{d}(n)$ for which $w(a)=w(v)$ are strongly associated with $v$. Even though there might be other spikes of degree $d$ which do not satisfy the hypothesis of the lemma, one may all the same obtain partial results for $\operatorname{dim}\left(\mathbf{Q}^{d}(n)\right)$ by applying an inductive procedure on $n$ as above to compute the dimension of the subspace of $\mathbf{Q}^{d}(n)$ generated by monomials in the weight class of $v$.

In [19] one such case is considered. The spike $v=$ $x_{1}^{2^{\lambda}-1} \cdots x_{n-1}^{2^{\lambda}-1}$ of degree $d(\lambda)=(n-1)\left(2^{\lambda}-1\right)$ satisfies the hypothesis of Lemma 11 and is one for which $\lambda_{1}-\lambda_{n-1}=0$. It is shown that if $\lambda \leq n-1$, then the dimension of the subspace of $\mathbf{Q}^{d(\lambda)}(n)$ generated by monomials in the weight class of $v$ is equal to $n+\sum_{q=2}^{\lambda}\left(\begin{array}{l}n \\ q\end{array}\right)$ and that if $\lambda \geq n$, then the dimension of the respective subspace is $2^{n}-1$.

\section{Disclosure}

This work was done while the first author was visiting the African Institute for Mathematical Sciences (AIMS).

\section{Conflict of Interests}

The authors declare that there is no conflict of interests regarding the publication of this paper.

\section{Acknowledgments}

The authors would like to thank AIMS and its director Professor Barry Green for their support and kind hospitality. Finally they would like to thank Sum for making a copy of his document [7] available to them.

\section{References}

[1] J. Adem, "The relations on Steenrod powers of cohomology classes," in Algebraic Geometry and Topology, A Symposium in Honor of S. Lefschetz, pp. 191-238, Princeton University Press, Princeton, NJ, USA, 1957.

[2] J. H. Silverman, "Hit polynomials and conjugation in the dual Steenrod algebra," Mathematical Proceedings of the Cambridge Philosophical Society, vol. 123, no. 3, pp. 531-547, 1998.

[3] W. M. Singer, "On the action of Steenrod squares on polynomial algebras," Proceedings of the American Mathematical Society, vol. 111, no. 2, pp. 577-583, 1991.

[4] R. M. Wood, "Steenrod squares of polynomials and the Peterson conjecture," Mathematical Proceedings of the Cambridge Philosophical Society, vol. 105, no. 2, pp. 307-309, 1989.

[5] M. Kameko, Products of projective spaces as Steenrod modules [Ph.D. thesis], John Hopkins University, Baltimore, Md, USA, 1990.

[6] M. Kameko, Generators of the Cohomology of $B V_{4}$, Toyama University, Toyama, Japan, 2003. 
[7] N. Sum, The Hit Problem for the Polynomial Algebra of Four Variables, University of Quy Nhon, Quy Nho'n, Vietnam, 2007.

[8] F. P. Peterson, "Generators of $H^{*}\left(R P^{\infty} \wedge R P^{\infty}\right)$ as a module over the Steenrod algebra," Abstracts of Papers Presented to the American Mathematical Society, vol. 833, pp. 55-89, 1987.

[9] T. N. Nam, “ $\mathscr{A}$-générateurs génériques pour l’algèbre polynomiale," Advances in Mathematics, vol. 186, no. 2, pp. 334-362, 2004.

[10] F. P. Peterson, “ $\mathscr{A}$-generators for certain polynomial algebras," Mathematical Proceedings of the Cambridge Philosophical Society, vol. 105, no. 02, pp. 311-312, 1989.

[11] W. M. Singer, "The transfer in homological algebra," Mathematische Zeitschrift, vol. 202, no. 4, pp. 493-523, 1989.

[12] N. Sum and D. V. Phuc, On a minimal set of generators for the polynomial algebra of five variables as a module over the Steenrod algebra [M.S. thesis], University of Quy Nhon, Quy Nhon, Vietnam, 2013.

[13] N. K. Tin, "The admissible monomial basis for the polynomial algebra of five variables in degree eight," Journal of Mathematical Sciences and Applications, vol. 2, no. 2, pp. 21-24, 2014.

[14] M. F. Mothebe, "Dimensions of the polynomial algebra $\mathbb{F}_{2}\left[x_{1}, \ldots, x_{n}\right]$ as a module over the Steenrod algebra," JP Journal of Algebra, Number Theory and Applications, vol. 13, no. 2, pp. 161-170, 2009.

[15] N. Sum, "On the Peterson hit problem of five variables and its applications to the fifth Singer Transfer," East West Journal of Mathematics, vol. 16, no. 1, pp. 47-62, 2014.

[16] G. Walker and R. M. W. Wood, "Young tableaux and the Steenrod algebra," in Proceedings of the School and Conference in Algebraic Topology, (Hanoi, 2004), vol. 11, pp. 379-397, Geometry and Topology Monographs, 2007.

[17] M. Kameko, "Generators of the cohomology of $B V_{3}$," Journal of Mathematics of Kyoto University, vol. 38, no. 3, pp. 587-593, 1998.

[18] N. Sum, “The negative answer to Kameko's conjecture on the hit problem," Advances in Mathematics, vol. 225, no. 5, pp. 23652390, 2010.

[19] M. F. Mothebe, "Dimension result for the polynomial algebra $\mathbb{F}_{2}\left[x_{1}, \ldots, x_{n}\right]$ as a module over the Steenrod algebra," International Journal of Mathematics and Mathematical Sciences, vol. 2013, Article ID 150704, 6 pages, 2013. 


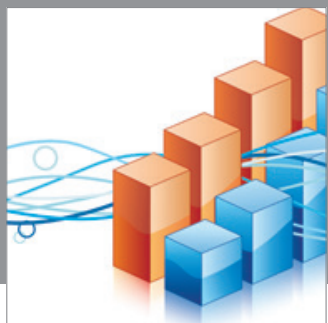

Advances in

Operations Research

mansans

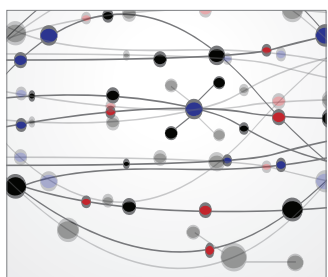

The Scientific World Journal
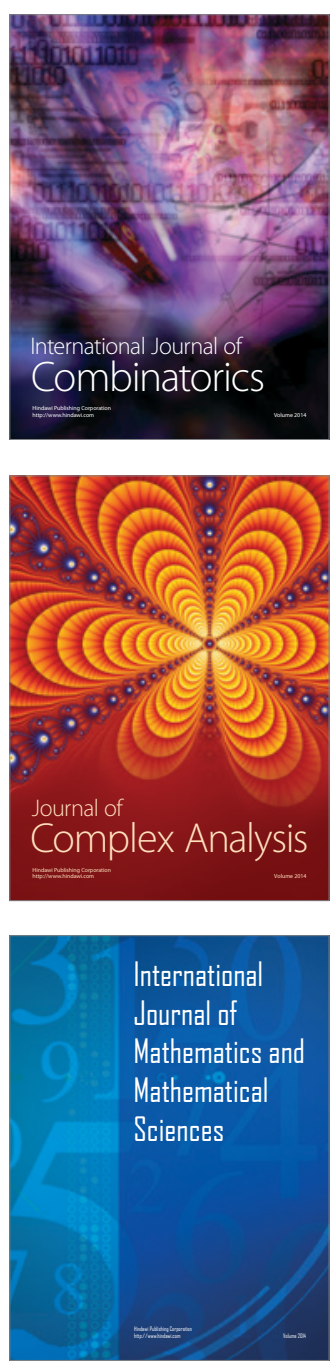
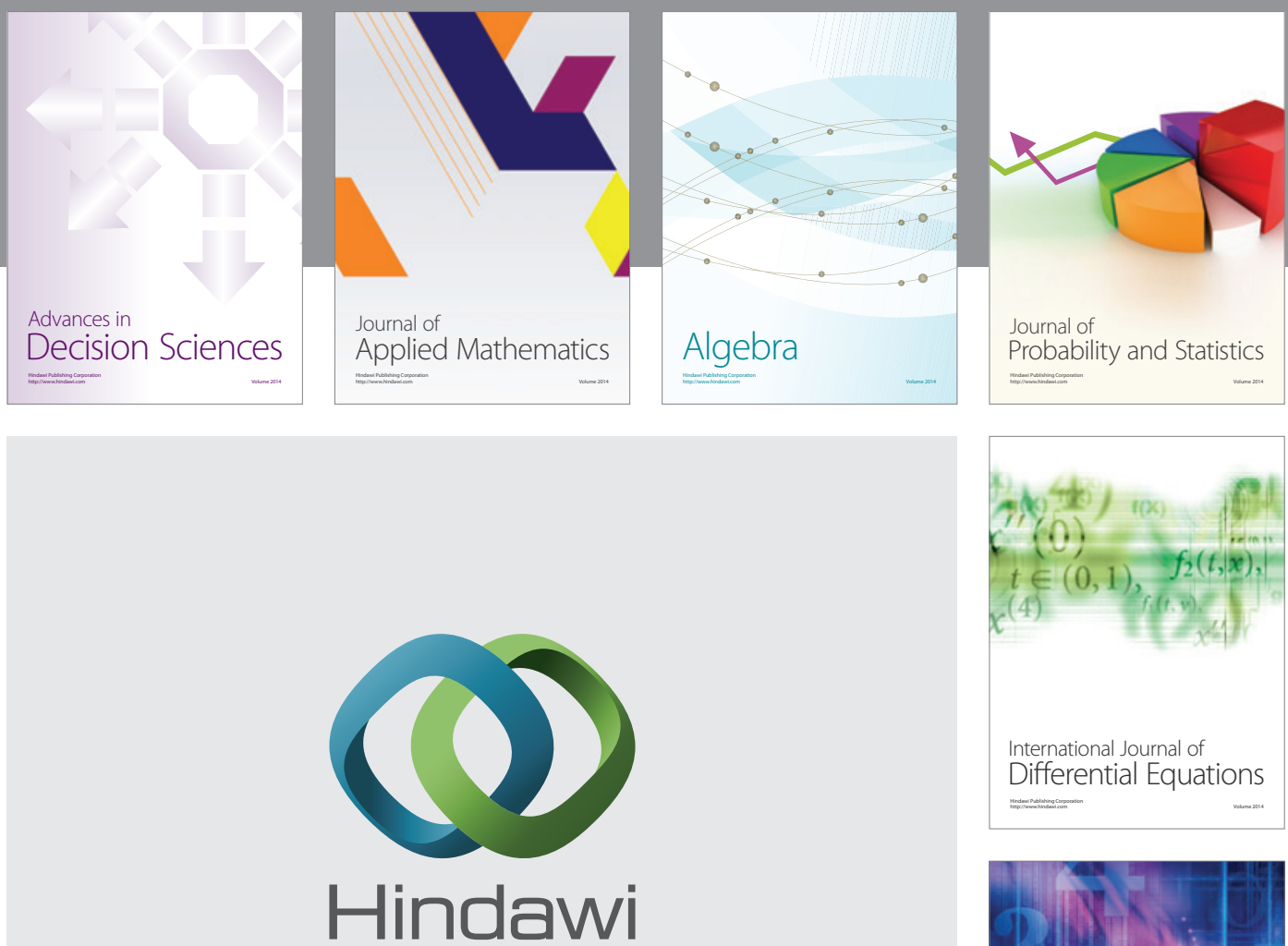

Submit your manuscripts at http://www.hindawi.com
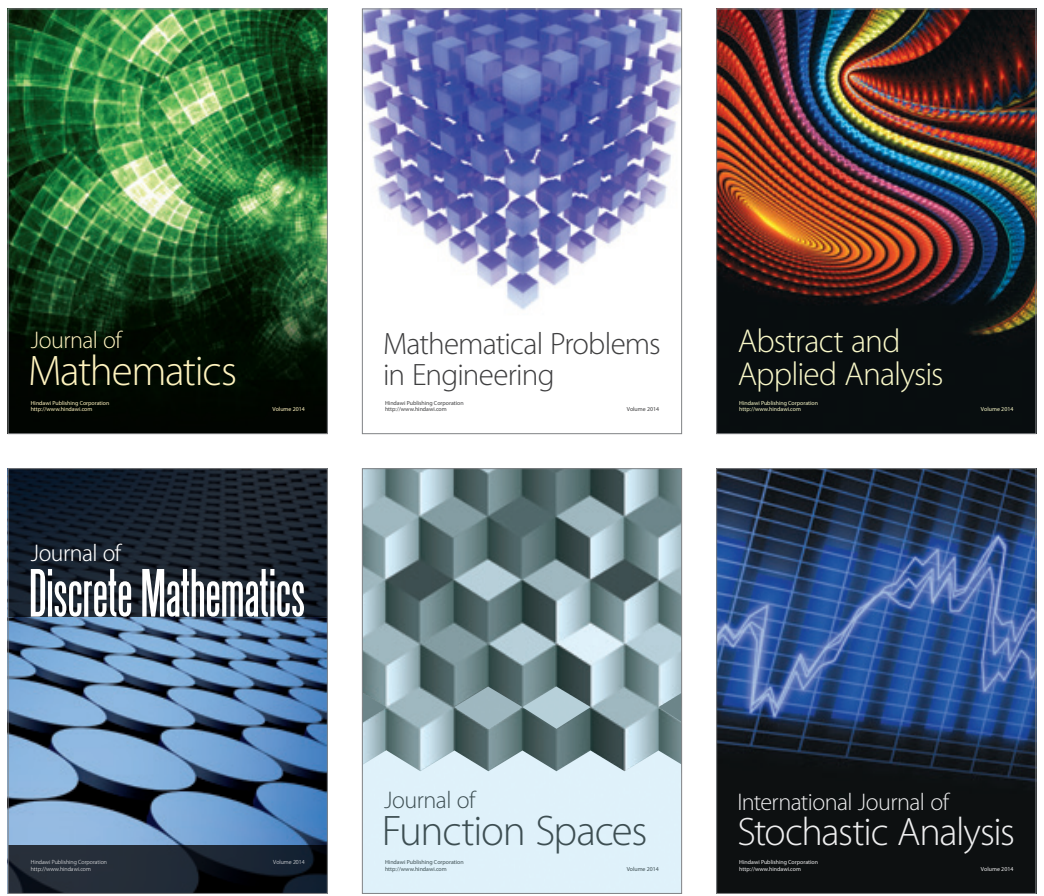

Journal of

Function Spaces

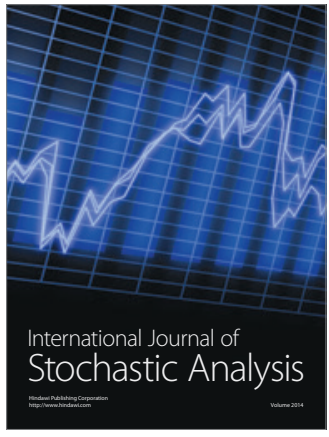

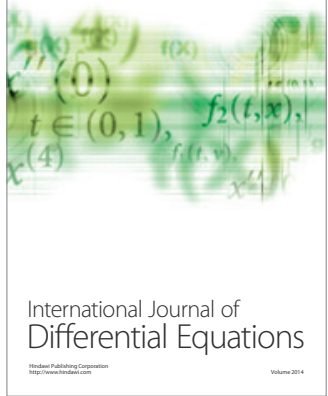
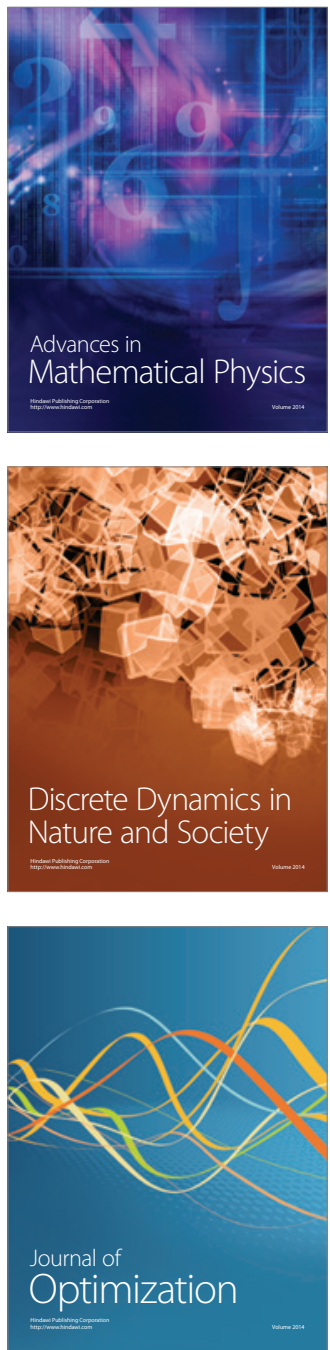